defects of skin or mucous membrane, care must be taken to include only the skin or mucous membrane proper without any fat; and usually a piece is transplanted three-sixteenths inch by one-fourth inch and always transferred at once to its final resting-place on a fresh, raw or healthy granulating surface, and after being stcured in position is not disturbed for at least three days. Perfect draining opportunities must, however, be provided, and strict aseptic precautions must be maintained throughout. If only the epithelial layer is used as a graft the resulting cicatrix is feeble, and from slight irritation the surface recently closed is likely to lose all the protection the physician was able to procure in weeks of patient toil.

CASE 3.-In March of this year a little girl 7 years of age was referred to me by Dr. Tobin of Mt. Sterling, Iowa, for cicatricial contraction and flexion, to an extreme degree, of the ring and little fingers of the right hand from a burn produced eight months before by a rope being drawn rapidly through the hand. Previous experience had convinced me of the folly of attempting to cure the case while the mass of cicatrix remained. I carefully removed all the scar tissue, at points exposing the flexor sublimus and profundus tendons, until the fingers could be easily and perfectly straightened, except a slight defect at the last joint of the little finger. The entire palmar surface of each finger was denuded. Two pieces of skin were next carefully removed from the forearm of the same limb of sufficient size to nicely cover the defects after allowance was made for shrinkage. The pieces removed were one-third by two and one-half inches. These large pieces or grafts were carefully sutured in place with fine silkworm gut tailings, as I could not trust a child of her age to leave the dressings entirely undisturbed. The hand was kept wrapped in an abundance of cotton and resting for six days and nights on a hot-water bottle at 125 Fahrenheit. Union was complete throughout on the little finger. Slight slough took place in the center of the ring finger flap, but not enough to be serious. The fingers were kept extended by splint for three weeks. The tendency to contraction was very slight indeed when last reported. This is the first case in which I have attempted to transplant bodily so large a piece of skin, and the first in which I know of sutures being used to retain a graft.

The plastic face cases are by far the heaviest and most extensive I have undertaken, and the worst I know to have been closed by any form of plastic procedure.

\section{A NEW DRY SURGICAL DRESSING.}

\section{ALBERT C. BARNES, A.M., M.D.}

Late of Pharmacological Institute, University of Heidelberg: $\mathbf{M} \mathrm{em}$ ber of Americau Therapeutic Society. AND

HERMANN HILLE, PH.D.

Late of Chemical Institute, Heidelberg; Formerly Assistant, Physiological Institute, Würzburg. PIIILADELPIIIA.

Whatever interest the manifold substitutes for iodoform may have for the surgeon or dermatologist, they are too similar in chemical construction and attributes to claim the serious attention of the scientific pharmacologist familiar with the laws of the relationship between chemical constitution and physiologic activity; the latter knows that clinically they must be of practically identical worth and have all the same limitations, disadvantages or dangers. Surgeons employ the odoriferous, toxic, non-antiseptic iodoform in the treatment of infected wounds because they know that not one of the substitutes is of equal value in cleaning the wound of the products of septic and necrotic processes and in stimulating healthy granulation. Aside from the stigmatizing odor of iodoform, the need of a substitute therefor has received additional emphasis by the fact that within comparatively recent times surgeons everywhere have frequently recorded more or less severe forms of headache, with or without anuria or albuminuria. which were clearly ascribable to the local use of but moderate quantities of iodofarm.

Practically all of the iodoform substitutes thus far proposed belong to one or the other of two classes and have, therefore, certain advantages and disadvantages. The first group comprises those which, like iodoform. depend for their activity upon the presence of iodin loosely combined chemically so that free iodin is eliminated by contact with the wound secretions. The best known of these is aristol (dithymol diodid), which is of certain value in a limited number of cases but which is too easily decomposed and too expensive to employ even if it fulfilled the requirements of a satisfactory dressing. Clinically the antiseptic and granulation-stimulating properties of free iodin are desirable. but there are other striking indications for treatment in infected wound surfaces which are not met by these iodin-bearing powders.

To the second group of iodoform substitutes belong the large number of bismuth compounds, depending for their activity upon bismuth which. as is well known, acts as a protective and diminishes secretions. Kocher of Bern employed and recommended bismuth subnitrate as a dressing for wounds attended with pus formation and excessive secretions. Hans Meyer of Marburg showed that the drying effect of bismuth is due to the mechanical plugging of the blood and lymph capillaries by minute particles of bismuth and that, anatomically considered, the effect is analogous to the healing which takes place under a scar. The newer organic bismuth compounds are of value clinically because they diminish wound secretions in the manner above indicated, but are possessed of but feeble, if any, antiseptic power and have no stimulating effect upon granulation; this is true not only of dermatol (subgallate) but also of the host of other bismuth compounds that have been offered under fanciful names.

By application of the newest procedures of synthetic chemistry, the writers have endeavored to produce a substitute for jodoform that would chemically, pharmacologically and clinically, possess the attributes which the teachings of modern surgery assert are necessary to meet the indications for treatment in infected wounds. Such a powder should be antiseptic, astringent, sedative. desiccating, stimulating to granulation and non-toxic. From a chemical standpoint the first step is to combine, synthetically, bodies which will gradually dissociate when brought in contact with wound secretions and thereby unfold their chemical and physiologic effects. We produced a series of compounds, all entirely new definite chemicals, and studied their chemical, pharmacologic and clinical properties. The essential features of one of these compounds, monoiodid-di-bismuth-methylene-dicresotinate produced for the first time by ourselves, are herewith briefly presented. This body is synthesized from cresotinic acid, formaldehyde, bismuth hydroxid and iodin in the following manner: Creosotinic acid, the least toxic and strongest antiseptic of the phenol group, is treated with formaldehyde and the thereby resulting methylene-dicresotinic acid is chemically com- 
bined witn iodin and bismuth hydroxid. This yields monoiodid-di-bismuth-methylene-di-cresotinate which has the structural formula

$$
\mathrm{CH}_{2} . \mathrm{C}_{6} \mathrm{H}(\mathrm{OH})\left(\mathrm{CH}_{3}, \mathrm{COOH} . \quad \mathrm{Bi} \stackrel{\mathrm{I}}{\mathrm{OH}}^{\mathrm{OH}}\right.
$$

This body is a pink, impalpable, odorless, tasteless and insoluble powder containing 45 per cent. of bismuth, 15 per cent. of iodin and 3 per cent. of formaldehyde. It is a well-known chemical law that when iodin and formaldehyde are combined, as they are in this body, they are dissociated in statu nascendi from their combination under the chemical and physical conditions present on wound surfaces. This evolution of free iodin and free formaldehyde takes place gradually, as can be easily demonstrated. The effects of this powder on a wound are those of bismuth, iodin, formaldehyde and cresotinic acid, i. e., antiseptic, astringent and alterative. The clinical use of the powder was preceded by bacteriologic tests and experiments on animals. Administered to dogs in dases of two grammes (30 grains) three time daily, it prnduced no toxic symptoms; the only effect noted was the production of constipation. The organisms of pus, as well as cultures of colon and typhoid bacilli, kept at $40 \mathrm{C}$. for from three hours to two days in contact with the powder, show no growth upon transplantation to fresh culture media; this fact illustrates the long-continued antiseptic power due to the gradual splitting off of the constituents.

For six months past the powder has been in constant daily use in several hospitals as a dressing after operations and in the general class of out-patient surgical cases in which are present active inflammatory processes accompanied by disorganization of tissue and excessive discharges. In the post-operative cases. union by adhesion or first intention was obtained in every case. Its use in infected wounds (burns, scalds, abscesses, suppurating surfaces, leg ulcers, etc.) showed remarkable effects in checking pus formation, drying secretions and in promoting granulations. In the out-patient surgical department of the Pennsylvania Hospital where the powder has been tried side by side with iodoform, aristol and several other dusting powders, it was noted that it uniformly cleans a wound better than any of the others, has an equal if not greater influence on granulation and induces more rapid healing. In no case have toxic effects of any kind resulted nor has it been necessary to discontinue its use because of disagreeable symptoms. This powder is interesting from a purely scientific standpoint because it is a new synthetic compound; clinically it is interesting because it is a compound of bismuth. iodin and formaldehyde so combined chemically that its active constituents are slowly split off, so that their effects are long drawn out and are not irritating or toxic. A detailed clinical report of the cases treated with the powder will be published later.

Ice or Heat for Local Application.-Memorabilien quotes Ewart to the effect that he has frequently found ice effective in painful articular rheumatism, arthritis and other affections which were aggravated or at least not improved by the application of heat. He rubbed the aching part with a smooth piece of ice and found that this gentle massage and cold cured a number of cases of acutely painful rheumatic arthritis of the hip-joint which had resisted all medicinal treatment and even baths of hot air. He also found that it relieved at once the severe pleuritic pains at the base of the lung in a number of cases of acute pneumonia.
NEW METHOD OF ANCHORING THE KIDNEYA PRELIMINARY REPORT.

BYRON B. DAVIS, M.D.

Surgeon to Immanuel Hospital, Professor of Principles of Surgery and Clinícal Surgery, Omaha Medical College, etc.

OMAHA, NER.

If a multiplicity of methods is an indication of unsatisfactory results, nephropexy must be the least satisfactory operation known to the surgeon. Dozens of methods have been exploited, each with a claim of superiority, but all based on four cardinal principles.

1. In April, 1881, Hahn reported his first cases of nephrorraphy for movable kidney. The sutures were passed through the fibrous capsule proper, the kidney being fastened to the edges of the deeper structures of the lumbar wound. Many slight modifications of this method have been made, but the principle of all has been the same as that originally used by Professor Hahn.

2. The method of decortication. Tuffier was the first to practice this method. He dissects off a portion of the posterior surface and convex border of the proper capsule of the kidney, and the organ is held into the wound by sutures, thus obtaining direct union between the deep muscles and fascia and the parenchyma. Jacobson modified this operation by dissecting off a flap of the fibrous capsule and stitching it to the lumbar fascia and muscles. Others make a double flap of proper capsule and suture each fiap to the respective wound edges.

3. Senn's ingenious method, by which the kidney is held in position by a sling of gauze about the upper and lower poles, and the whole wound allowed to heal by granulations, is now one of the recognized principles of treatment of this troublesome disease.

4. Vulliet's method of making use of a strip from the tendon of the erector spinæ muscle to underrun the fibrous capsule, the free end of the strip of tendon being then attached to thr muscle, illustrates a fourth principle in the treatment.

\section{DISAPPOINTMENT FROM ALL METHODS.}

Surgeons have been much disappointed in the results of all these methods. Relapses are frequent and the more carefully one follows up the after history of his cases, the less confidence he is likely to have in his results. I know of some of my cases that have relapsed and have been impressed with the number of cases that have come to me with freely movable kidneys and bearing the sear of a previous operation done by well-known skilful surgeons. The adhesions between the true capsule and the lumbar structures are not likely to be firm. They become stretched in time and the kidney is likely to become as movable as before. The same may be said of the Senn method. No matter how firmly the kidney is held in place by the cicatricial tissue at first, we all know the later history of cicatricial tissue and there is no reason to suppose that in this region it will behave in any different manner than usual. All know the fate of large numbers who were subjected to the McBurney operation for hernia a few years ago, the plug of cicatricial tissue acting well for a time, but finally softening and stretching, allowing relapse of the hernia. Another theoretical objection to the Senn method is the effect of so much scar tissue upon so delicate an organ as the kidney.

Flaps of the true capsule stitched into the wound have appealed to me as being the best of the tried methods. But even here we have to trust to the adhesion between capsule and wound structures remaining intact without 